\title{
Coherent optical focal plane array receiver for PPM signals: investigation and applications
}

M. Muñoz Fernández, K. M. Cheung, R. Mukai

M. Muñoz Fernández, K. M. Cheung, R. Mukai, "Coherent optical focal plane array receiver for PPM signals: investigation and applications," Proc. SPIE 6304, Free-Space Laser Communications VI, 63040I (1 September 2006); doi: $10.1117 / 12.681534$

SPIE. Event: SPIE Optics + Photonics, 2006, San Diego, California, United States 


\title{
Coherent ${ }^{1}$ Optical Focal Plane Array Receiver for PPM Signals: Investigation and Applications ${ }^{2}$
}

\author{
M. Muñoz Fernández, ${ }^{\mathrm{ab}}$ K.M. Cheung, ${ }^{\mathrm{b}}$ and R. Mukai ${ }^{\mathrm{b}}$ \\ Email: Michela.Munoz.Fernandez@jpl.nasa.gov, phone : (818)354 5046; fax : (818)393 6871 \\ ${ }^{a}$ California Institute of Technology, 1200 E. California Blvd., Pasadena, CA 91125 \\ ${ }^{\mathrm{b}}$ Jet Propulsion Laboratory, California Institute of Technology, 4800 Oak Grove Dr., Pasadena, \\ CA 91109
}

\begin{abstract}
The performance of a coherent optical focal plane array receiver for PPM signals under atmospheric turbulence is investigated and applications of this system are addressed. The experimental demonstration of this project has already been explained in previous publications [1,9]. This article shows a more exhaustive analysis of the expressions needed to obtain the Bit Error Rate (BER) for the real system under study in the laboratory, specifically an approach using the Saddle-Point approximation [10] of the MarcumQ function. Selected experimental results of this system are described and compared with theoretical BER expressions, and array combining gains are presented. Receiver sensitivity in terms of photons per bit (PPB) is examined; BER results are shown as a function of signal to noise ratios (SNR) as well as a function of photons per bit.
\end{abstract}

Keywords: focal plane array, coherent optical communications, shot noise limited performance, SaddlePoint approximation.

\section{Introduction}

In clear weather there are random, time-varying patterns in the index of refraction of the air in the Earth's atmosphere. When a laser signal from a distance spacecraft (assumed to be a plane wave due to the tremendous distances involved in deep space communications) passes through the atmosphere, the differences in refractive index will distort the planar phase front of the incoming signal wave. This causes the wavefront that reaches the receiving telescope aperture to suffer from significant phase distortions that will cause the received signal pattern in the receiver focal plane to be spread out and to be significantly distorted. A single small detection element designed to capture the Airy pattern resulting from a perfect plane wave reaching the aperture will be too small to catch most of the signal, resulting in severe signal losses. One could attempt to compensate for the spreading of the signal in the detector focal plane by using a single very large receiving element in the focal plane. However, the field in the focal plane is a complex field with both magnitude and phase. A single large detector element will effectively perform a simple

\footnotetext{
1 "Coherent" here means mixing the incoming optical signal with a locally generated local oscillator laser to produce an intermediate frequency (IF) signal that can be demodulated and processed using traditional methods.

2 The research described in this publication was carried out at the Jet Propulsion Laboratory, California Institute of Technology, under a contract with the National Aeronautics and Space Administration.
} 
addition of the focal plane field over its surface, and this could easily result in destructive signal cancellation, again resulting in severe signal losses. The key to capturing the scattered, phase and amplitude distorted signal field in the focal plane is to have an array of small elements. As signal components of varying amplitude and phase hit the array, the outputs of the receiving elements are coherently ${ }^{3}$ added together in order to reconstruct the original signal, avoiding both the self-cancellation that often occurs with large detection elements and the waste of useful signal energy in the focal plane that results from having a single small detection element incapable of capturing the entire signal field.

\section{Probability of bit error for non-coherent (heterodyne) optical receiver with random phase channels for pulse position modulated (PPM) signals}

The equipment used in the lab had some limitations and constraints, necessitating a mathematical model that would take these constraints into account. The PPM modulator is not ideal, and during the dead time it leaks signal energy. This unwanted signal energy, referred to as "leakage," was mixed with the local oscillator laser, generating a heterodyned beatnote in the noise slots. This resulted in a Gaussian noise with non-zero mean in the noise slots, which in this section will be explained in detail. Different channels presented different leakage characteristics that have been taken into account in the following signal model based on a non-central chi square distribution for the signal as well as the noise slots. In this section, expressions for the BER in the presence of leakage are derived, and the case in which leakage is not present is also examined.

First, the matched filter output in the signal slot is taken under consideration. Since the signal consists of two Gaussian random variables with non-zero mean in the real and imaginary parts, the resulting probability distribution function (pdf) is a non-central chi-square pdf with two degrees of freedom. Let $m_{1}$ and $m_{2}$ be the means of the real and imaginary parts of the complex Gaussian, and let them both have variance $\sigma^{2}$. Define the sum of the squares of the means:

$$
s^{2}=m_{1}^{2}+m_{2}^{2}
$$

Then the pdf of signal magnitude $\mathrm{Y}$ is given by:

$$
f_{Y, \text { sig }}(y)=\frac{1}{2 \sigma^{2}} \exp \left[-\frac{y+s^{2}}{2 \sigma^{2}}\right] I_{0}\left(\frac{s \sqrt{y}}{\sigma^{2}}\right)
$$

where $I_{0}(x)$ is the zeroth order modified Bessel function of the first kind.

Consider the case when a leakage term is present in the noise slots, and the sum of squared means of the real and imagery parts of the complex Gaussian is for those slots, $s_{L}^{2}$, which is non-zero. The resulting cumulative distribution function (cdf) of the noise magnitude $\mathrm{Y} \leq y$ is given by:

\footnotetext{
3 "Coherently" here means aligning signals in phase.
} 


$$
\begin{aligned}
F_{Y, \text { noise }}(Y \leq y) & =1-Q_{1}\left(\frac{s_{L}}{\sigma}, \frac{\sqrt{y}}{\sigma}\right) \\
& =1-\int_{\frac{\sqrt{y}}{\sigma}}^{\infty} x \exp \left(-\frac{x^{2}+\left(\frac{s_{L}}{\sigma}\right)^{2}}{2}\right) I_{0}\left(\frac{s_{L}}{\sigma} x\right) d x
\end{aligned}
$$

where $Q_{1}(a, b)=\int_{b}^{\infty} x \exp \left(-\frac{x^{2}+a^{2}}{2}\right) I_{0}(a x) d x$ is the Marcum-Q function. A correct decision is made when the signal magnitude $y$ is higher than everyone of the noise magnitude in the $M-1$ noise slots. Thus integrating all possible value of $y$ the probability of a correct decision $P(C)$ is given by

$$
P(C)=\int_{0}^{\infty} f_{Y, \text { sig }}(y)\left(F_{Y, \text { noise }}(Y \leq y)\right)^{M-1} d y .
$$

Plugging Eqs. (2) and (3) into Eq. (4) the probability of a correct decision is as follows:

$$
P(C)=\frac{1}{2 \sigma^{2}} \int_{0}^{\infty} \exp \left[-\frac{y+s^{2}}{2 \sigma^{2}}\right] I_{0}\left(\frac{s \sqrt{y}}{\sigma^{2}}\right)\left(1-Q_{1}\left(\frac{s_{L}}{\sigma}, \frac{\sqrt{y}}{\sigma}\right)\right)^{M-1} d y
$$

The integral in Eq. (5) presents the following difficulties:

1. It does not have a known closed form expression.

2. It is difficult to evaluate numerically for large $M$ (i.e., $M=256$ ).

One approach is to upper bound and lower bound the Marcum-Q function, and by using numerical bounds judiciously it is possible to obtain upper and lower bounds for the probability of symbol error given in Eq. (5). The bounds on the Marcum-Q function are given by [8];

$$
\exp \left[-\frac{(b+a)^{2}}{2}\right] \leq Q_{1}(a, b) \leq \exp \left[-\frac{(b-a)^{2}}{2}\right]
$$

The bounds in Eq. (6) play an important role in the analysis of the bit error probability performance of this system. Another possibility involves direct approximation of the integral in Eq. (5) which we will show in the later part of this section.

\section{Expressions specific to the case without leakage}

Below a series of expressions specific to the case without leakage are given. If there is no leakage, then $s_{L}=0$ and the cdf $F_{Y, n o i s e}(Y \leq y)$ is then given by: 


$$
F_{Y, \text { noise }}(Y \leq y)=1-Q_{1}\left(0, \frac{\sqrt{y}}{\sigma}\right)
$$

This makes the upper and lower bounds in Eq. (6) equal to each other and therefore tight. It is now possible to write:

$$
F_{Y, \text { noise }}(Y \leq y)=1-\exp \left(-\frac{y}{2 \sigma^{2}}\right)
$$

The resulting expression for symbol error probability $P(E)=1-P(C)$ is:

$$
1-\frac{1}{2 \sigma^{2}} \int_{0}^{\infty} \exp \left[-\frac{y+s^{2}}{2 \sigma^{2}}\right] I_{0}\left(\frac{s \sqrt{y}}{\sigma^{2}}\right)\left(1-\exp \left(-\frac{y}{2 \sigma^{2}}\right)\right)^{M-1} d y
$$

which is equivalent to the expression derived in [6] for $M$-ary orthogonal symbol error probabilities for non-coherent (from a communications perspective) receivers. Since:

$$
\frac{E_{S}}{N_{0}}=\frac{s^{2}}{2 \sigma^{2}}
$$

is the SNR, it is possible to perform a change of variables in order to obtain symbol error probability as a function of SNR. The final answer in the no leakage case is [6]:

$$
P(E)=\sum_{m=1}^{M-1}\left((-1)^{m+1} \frac{1}{m+1}\left(\begin{array}{c}
M-1 \\
m
\end{array}\right) \exp \left(-\frac{m}{m+1}\left(\frac{E_{S}}{N_{0}}\right)\right)\right)
$$

For the case of signals under Additive White Gaussian Noise (AWGN), the probability of bit error is given by the same expression from Eq. (2), but instead of using the SNR of one channel, the right term to use is the addition of the SNR of the individual channels to be combined [7]. Expressing the SNR directly as a ratio instead of in decibels and letting $\left(\frac{E_{S}}{N_{0}}\right)_{i}$ denote the SNR of the $i^{\text {th }}$ channel, the total SNR is:

$$
\left(\frac{E_{S}}{N_{0}}\right)_{\text {total }}=\sum_{i=1}^{N}\left(\frac{E_{S}}{N_{0}}\right)_{i}
$$

for the case of $N$ channels. Eq. (12) assumes that the noise is zero-mean uncorrelated Gaussian.

\section{Expressions specific to the case with leakage}

Since even numerical evaluation of the integral for the symbol error probability in (5) is difficult in general, a different strategy was taken. An upper bound on $Q_{1}(a, b)$ yields an upper bound on the probability of making a symbol error, and similarly a lower bound on $Q_{1}(a, b)$ yields a lower bound on the symbol error probability. The upper bound expression is given by [9]: 


$$
P_{\text {upper }}(E)=1-\frac{1}{2 \sigma^{2}} \int_{0}^{\infty} \exp \left[-\frac{y+s^{2}}{2 \sigma^{2}}\right] I_{0}\left(\frac{s \sqrt{y}}{\sigma^{2}}\right)\left(1-\exp \left(-\frac{\left(\sqrt{y}-s_{L}\right)^{2}}{2 \sigma^{2}}\right)\right)^{M-1} d y
$$

Similarly, the lower bound expression is:

$$
P_{\text {lower }}(E)=1-\frac{1}{2 \sigma^{2}} \int_{0}^{\infty} \exp \left[-\frac{y+s^{2}}{2 \sigma^{2}}\right] I_{0}\left(\frac{s \sqrt{y}}{\sigma^{2}}\right)\left(1-\exp \left(-\frac{\left(\sqrt{y}+s_{L}\right)^{2}}{2 \sigma^{2}}\right)\right)^{M-1} d y
$$

The bounds on SER given by Eqs. (13) and (14) were evaluated via numerical integration using measured values of the following three parameters:

1. $s^{2}:$ The sum of the squared means of the matched filter outputs in a signal slot.

2. $s_{L}^{2}$ : The sum of the squared means of the matched filter outputs in a noise slot.

3. $\sigma^{2}$ : The variance of the matched filter real and imaginary parts. Since the real and imaginary parts each have variance $\sigma^{2}$, the total variance of the complex Gaussian matched filter output is $2 \sigma^{2}$.

The three parameters above must be estimated from the experimental data since the leakage is unknown a priori. It can be shown that Eqs. (13) and (14) can be easily computed numerically. The

comparisons between the upper bound, lower bound, and the empirical data are shown in figures 2,3 , and 4.

\section{Computing Bit Error Rate of Non-Coherent Detection of M-ary Orthogonal Signal in the Presence of Gaussian Noise with Non-Zero Mean Using Saddle-Point Approximation}

In the previous section, simpler upper and lower bounds of Eq. (5) were analyzed, but these bounds are loose for the high signal-to-noise ratio region. In this section the objective is to evaluate the Saddle-Point approximation [10] of the Marcum-Q function which in turn provides a simplified approximation of the BER expression of Eq. (5).

The Marcum-Q function is given by the following expression

$$
Q_{1}(a, b)=\int_{b}^{\infty} x e^{-\frac{x^{2}+a^{2}}{2}} I_{0}(a x) d x
$$

We define

$$
\begin{aligned}
& f(x)=x e^{-\frac{x^{2}+a^{2}}{2}} I_{0}(a x), \\
& g(x)=\log _{e} f(x)
\end{aligned}
$$


Note that $f(x)$ is uni-modal; applying Newton's Method on the derivative of Eq. (16) or using other optimization procedure we can find the unique $x_{m}$ such that $f\left(x_{m}\right)$ is maximized. Next we evaluate the first and second derivatives of $g(x)$ as follows:

$$
\begin{gathered}
g^{\prime}(x)=\frac{1}{x}-x+\frac{a I_{1}(a x)}{I_{0}(a x)} \\
g^{\prime \prime}(x)=\frac{1}{2}\left(-2+a^{2}-\frac{2}{x^{2}}+a^{2} \frac{-2 I_{1}(a x)^{2}+I_{0}(a x) I_{2}(a x)}{I_{0}(a x)^{2}}\right)
\end{gathered}
$$

The Saddle-Point approximation $Q_{1} *(a, b)$ of $Q_{1}(a, b)$ is given by

$$
Q_{1} *(a, b)=c \int_{b}^{\infty} f\left(x_{m}\right) e^{\frac{g^{\prime \prime}\left(x_{m}\right)\left(x-x_{m}\right)^{2}}{2}} d x
$$

where $f(x)$ and $g^{\prime \prime}(x)$ are defined in Eq. (16) and (19) respectively, and $x_{m}$ is the value of $x$ that maximizes $f(x)$. Thus we show that the Marcum-Q function can be approximated by a definite integral of a Gaussian distribution as shown in Eq. (20) ( $c$ is a normalization factor). Note that $x_{m}, f\left(x_{m}\right)$, and $g^{\prime \prime}\left(x_{m}\right)$ are implicit and explicit functions of $a$. This approximation is particularly good when $a$, which represents $\frac{s_{L}}{\sigma}$ in Eq. (5), is reasonably large. Next denote $\sigma_{m}=-\frac{1}{g^{\prime \prime}\left(x_{m}\right)}, Q_{1}^{*}(a, b)$ can be rewritten as:

$$
Q_{1} *(a, b)=\int_{b}^{\infty} \frac{1}{\sqrt{2 \pi} \sigma_{m}} e^{\frac{-\left(x-x_{m}\right)^{2}}{2 \sigma_{m}}} d x
$$

and

$$
1-Q_{1} *(a, b)=\frac{1}{2}\left(1+\operatorname{Erf}\left[\frac{b-x_{m}}{\sqrt{2} \sigma_{m}}\right]\right)
$$

Replacing the term $1-Q\left(\frac{s_{L}}{\sigma}, \frac{\sqrt{y}}{\sigma}\right)$ in Eq. (5) with the approximation expression in Eq. (22), the approximation of $P(E)$ is given by

$$
P(E) \approx 1-\frac{1}{2 \sigma} \int_{0}^{\infty} \exp \left[-\frac{y+s^{2}}{2 \sigma^{2}}\right] I_{0}\left(\frac{s \sqrt{y}}{\sigma^{2}}\right)\left(\frac{1}{2}\left(1+\operatorname{Erf}\left[\frac{\frac{\sqrt{y}}{\sigma}-x_{m}}{\sqrt{2} \sigma_{m}}\right]\right)\right)^{M-1} d y
$$


It can be shown that $P(E)$ in Eq. (23) can be easily computed numerically. A general and more detailed treatment on approximating the Marcum-Q function and other relevant probability functions will be given in an upcoming paper.

\section{Results of the Coherent Optical Receiver Experiment (CORE)}

The experimental setup [1,9] consists of two Nd:YAG (YAG denotes yttrium aluminum garnet) lasers operating at $1064 \mathrm{~nm}$, whose outputs are aligned and combined on the surface of a 4 X 4 Fermionics InGaAs detector array. One of the lasers serves as a local oscillator, while the other simulates the received signal. The two lasers are operated at slightly different wavelengths, yielding a relatively stable differencefrequency tone of approximately $6 \mathrm{MHz}$ in the detected signal. In the presence of spatial distortions simulating atmospheric turbulence conditions, the difference-frequency tone is generally observable in several array elements simultaneously, but usually with different phases. In the current coherent combining experiment, each of the 16 outputs of the detector array is amplified, and input to a 16-channel dataacquisition assembly (using GaGe data-acquisition cards). The analog signals are digitized to 8 bits at a sampling rate of 25 megasamples per second (MSPS). The modulation format for the transmitted laser signal is PPM using an external Electro-Optic Modulator (Pockle cell). The resulting sample stream acquired with the GageScope is digitally downconverted to complex baseband. The resulting downconverted complex samples served as input to a least-mean-square (LMS) algorithm, [9] which was used to estimate the complex weights required to reconstruct the signal. The complex-weighted samples from each channel were then combined, in order to maximize the combined signal-to-noise ratio (SNR). A rotating predistorted plexiglass plate was incorporated into the experimental setup to simulate atmospheric turbulence.This signal laser beam is focused into the photodetector via the receiver lens.

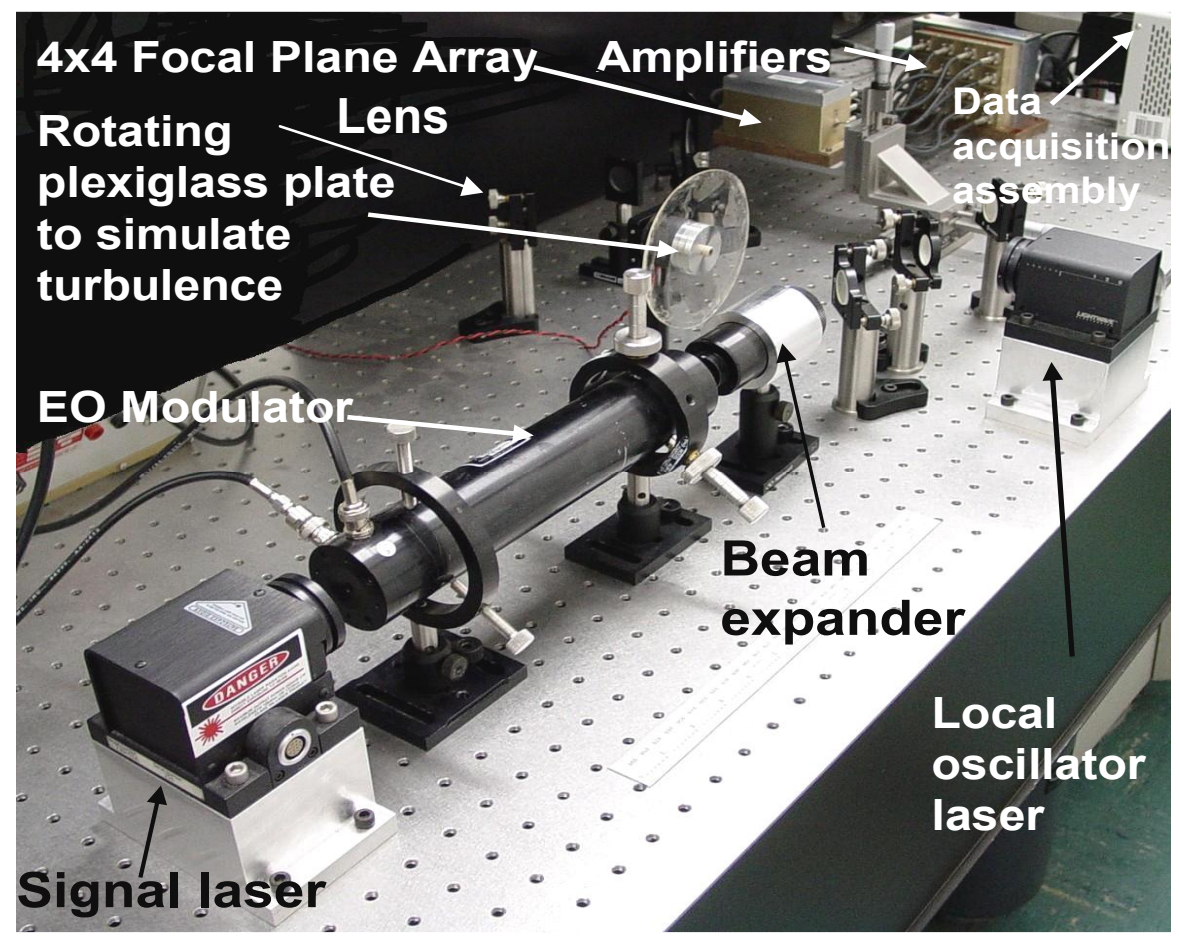

Figure 1. Coherent combining experiment at the Jet Propulsion Laboratory, NASA. 


\section{Evaluation of experimental system performance and comparison with theory}

A brief summary of the results will be shown in this section, and detailed results are available in $[1,9]$. Two configurations were used. In the first, a single detector with a high-quality transimpedance amplifier provided nearly shot-noise limited performance. In the second, an FPA detector was used. In

Figure 2, BER is plotted against photons per bit for the case of 256 PPM for a single detector configuration. The experimental points lie close to the theoretical BER performance line, and the trend line fitted to the experimental points is seen to lie very close to the theoretical bound. This figure shows a bit error rate only slightly above $3 \cdot 10^{-2}$ at 1 photon per bit, illustrating the system's performance in the presence of weak signals. Figure (3) shows the computation of hypothetical FPA performance if the detector elements had the same characteristics nearly shot-noise limited characteristics as the single detector [1,9]. In Figure (3), the number of photons per bit on the $\mathrm{x}$-axis refers only to the total number of photons per bit summed on all FPA channels and not to individual channel photon counts per bit.

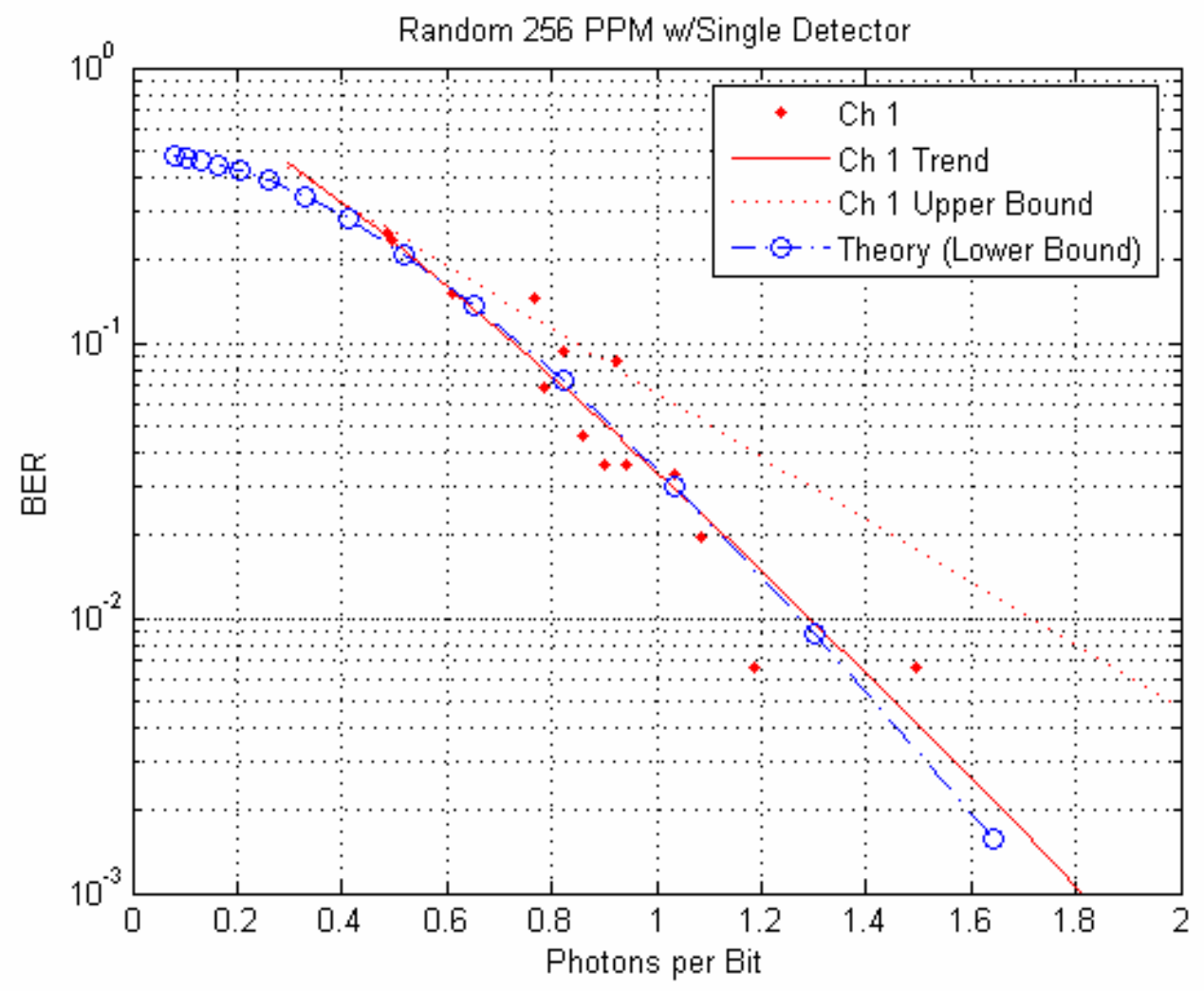

Figure 2. BER vs. photons per bit with the single detector (256 PPM). 


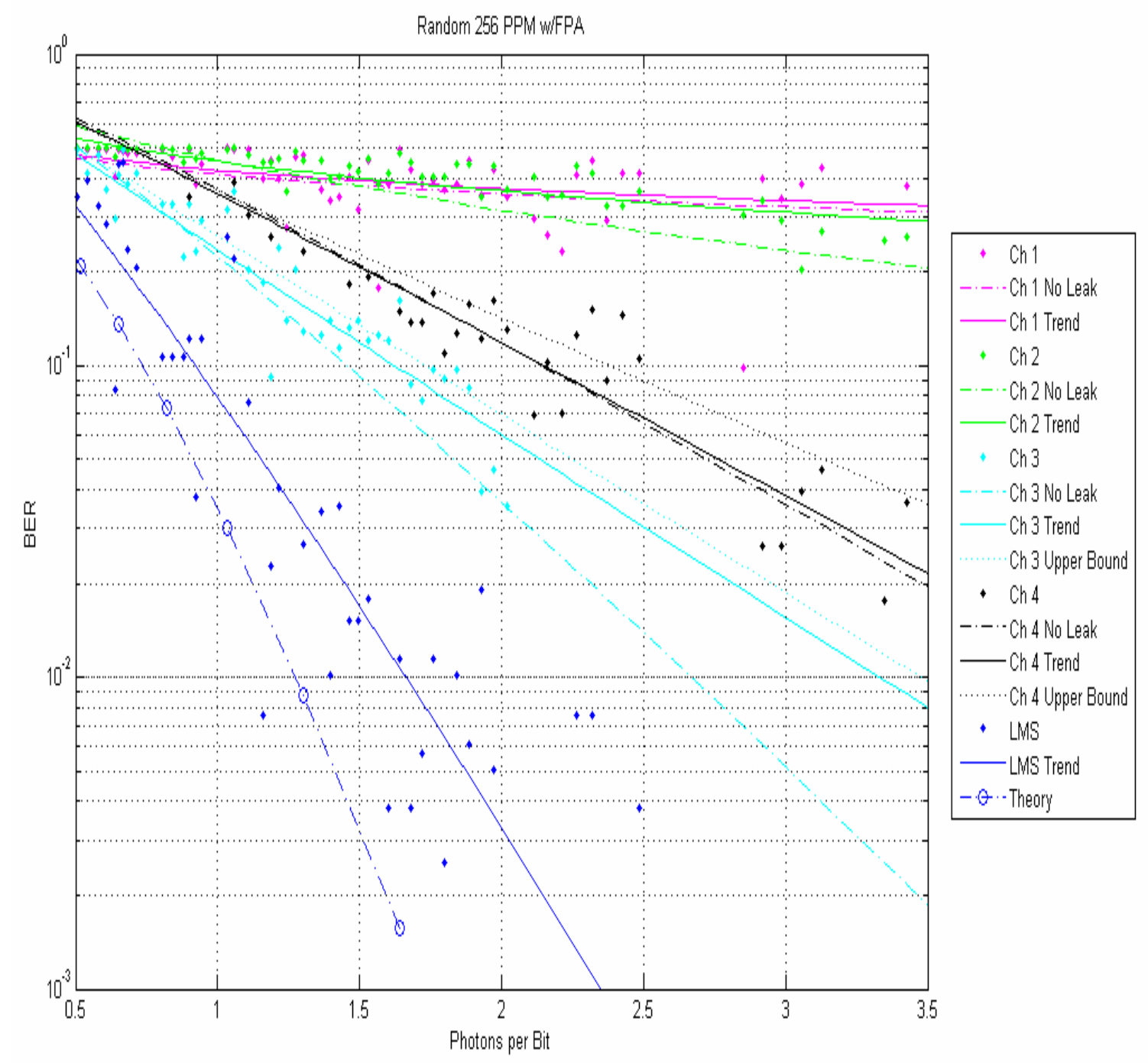

Figure 3. BER vs. photons per bit assuming an FPA with the same characteristics as the single detector (256 PPM). 


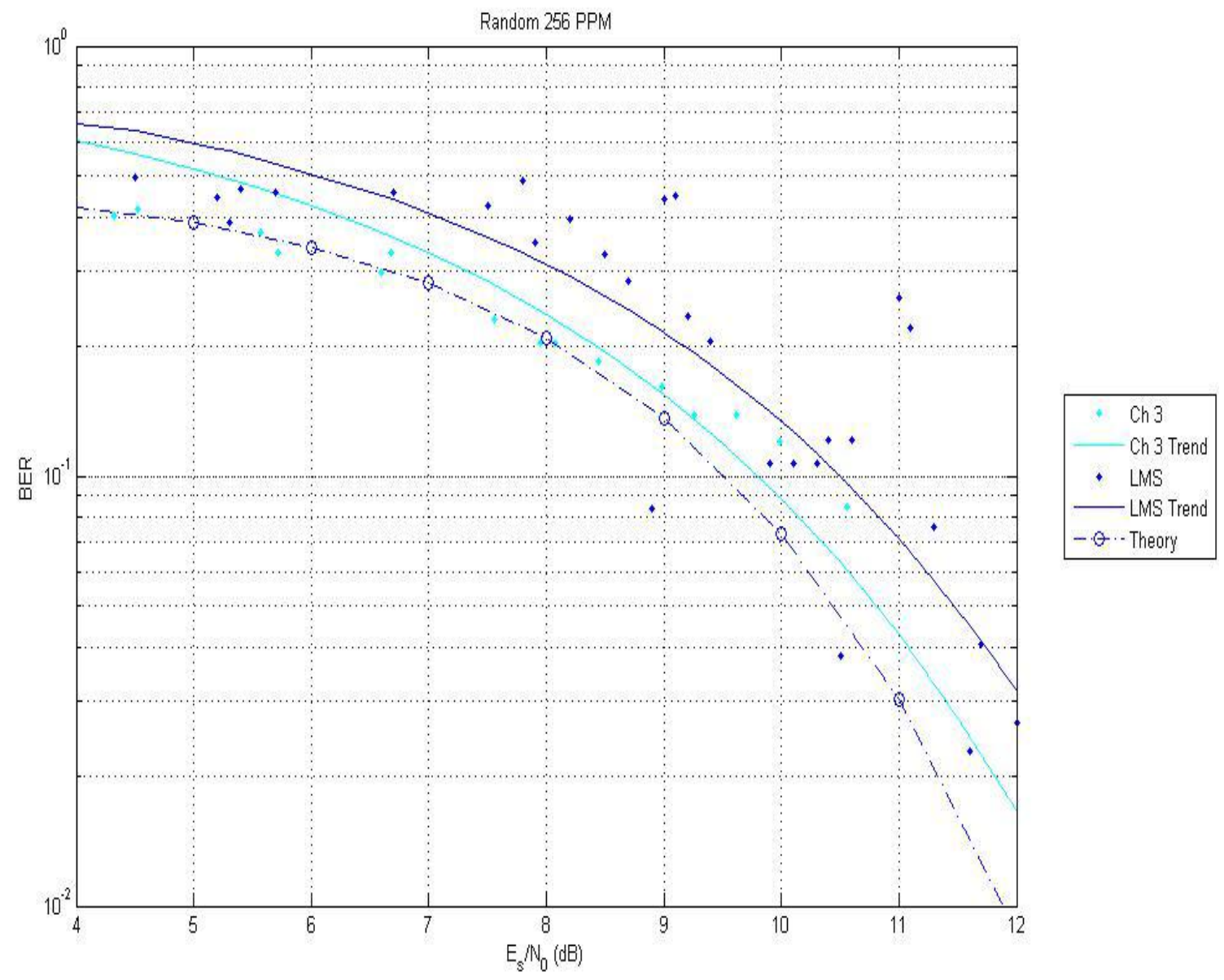

Figure 4. BER vs. Es/N0 for 256 PPM: Illustration of single channel performance vs. combined channel. Single channel BER is plotted vs. single channel SNR.

Figure (4) shows BER as a function of SNR for 256 PPM. Performance of a single channel (channel 3) is compared to that of the combined channel. It is important to notice that single channel BER is plotted vs. individual channel SNR and not against combined channel SNR as in Figure (3). One observes a $0.2 \mathrm{~dB}$ loss due to the leakage term in channel 3 illustrated by the horizontal difference between the theoretical (blue-dotted curve) and the experimental channel 3 curve (cyan). The combining loss for this case is 0.8 $\mathrm{dB}$ when comparing the experimental channel 3 curve with the experimental combined channel curve (continuous blue curve).

In summary, high quality low noise receiver electronics and/or a sufficiently powerful local oscillator laser operating at or near the shot noise limit, used in conjunction with a focal plane array, will enable bit error rate performance close to that achievable in the absence of atmospheric turbulence. Again, combined channel performance is within less than 0.5 photons per bit of the theoretical performance curve, indicating that much of the signal energy scattered across the five FPA elements used in combining can be recovered. These results indicate that coherently combining the outputs of the FPA channels permits very good 
reconstruction of the signal that would have existed if no turbulence had been present and if all photons had been hitting this detector.

\section{Conclusions and future directions}

Coherent combining algorithms for focal plane arrays are an area of further research. In a realistic space communications system utilizing PPM, the relative positions of the spacecraft and the ground receiver may change by many optical wavelengths between PPM pulses. Since no signal exists during the inter-pulse intervals, tracking of the spacecraft signal phase poses a challenge under some operating conditions, depending on the degree of pulse-to-pulse phase coherence present. The use of coding would unleash the true potential of the focal plane array receiver. Uncoded bit error rates below $10^{-1}$ are achievable using a shot-noise-limited receiver running at slightly less than one photon per bit. Corresponding coded bit error rates would be significantly lower, enabling very robust digital communications at low signal levels. The ability to communicate reliably at such weak signal levels would greatly extend the utility and usefulness of optical communications for deep space, especially in light of the severe demands generated by deep space link budgets.

Future work also includes comparing the resulting BER curves obtained with the Saddle-Point approximation of the Marcum-Q function with experimental results, upper bound and lower bound and compare those results with the main results obtained in this paper. Then, one could investigate how this result can apply to mobile communication scenarios to estimate BER of Rician channels and Nakagami-m fading model.

\section{REFERENCES}

1. M. Muñoz Fernández, V. A. Vilnrotter, R. Mukai, and Babak Hassibi, Coherent Optical Array Receiver Experiment: Design, Implementation and BER Performance of a Multichannel Coherent Optical Receiver for PPM Signals under Atmospheric Turbulence. Proc. of SPIE Vol. 6105R, (2006), FreeSpace Communication Technologies XVIII, ed. by G. Stephen Mecherle.

2. R. M. Gagliardi, S. Karp, Optical Communications, $2^{\text {nd }}$ ed., Wiley Series in Telecommunications and Signal Processing, New York, 1995, Chapter 6.

3. Larry C. Andrews, University of Central Florida, Field Guide to Atmospheric Optics, SPIE Field Guides, Volume FG02, John E. Greivenkamp, Series Editor, SPIE Press, Washington 2004.

4. J. W. Goodman. 2005. Introduction to Fourier Optics. Englewood, Colorado: Roberts \& Co.

5. S. Zohar, and V. Vilnrotter, "Using a Focal-Plane Array to Estimate Antenna Pointing Errors," JPL External Publication 91-45, October 1991.

6. M. K. Simon, S. Hinedi, and W. Lindsey. 1995. Digital Communication Techniques, Signal Design and Detection. Upper Saddle River, New Jersey: PTR Prentice Hall.

7. V. A. Vilnrotter, Rodemich, E.R.; Dolinar, S.J., Jr. March 1992. "Real-time combining of residual carrier array signals using ML weight estimates." IEEE Transactions on Communications, vol. 40, $\mathrm{n}$. 3, pp. 604-615.

8. M. K. Simon. 2002. Probability Distributions Involving Gaussian Random Variables, A Handbook for Engineers and Scientists. Norwell, Massachusetts: Kluwer Academic Publishers.

9. M. Muñoz Fernández, Coherent Optical Array Receiver For PPM Signals Under Atmospheric Turbulence, Ph.D. Thesis, Dept. of Electrical Engineering, California Institute of Technology, 2006.

10. David MacKay, "Information Theory, Inference, and Learning Algorithms," Cambridge University Press, 2005. 